\title{
Carrier gas flow arrangement based photoacoustic detection method for measuring gas permeability of polymer membranes
}

\author{
Zoltán Filus ${ }^{1}$, Nikolett Tóth ${ }^{2 *}$, Gábor Gulyás ${ }^{1}$, Tibor Guba ${ }^{2}$, Gábor Szabó ${ }^{2}$ and Zoltán \\ Bozóki ${ }^{2,3}$ \\ ${ }^{1}$ Hilase Development, Production, Service and Trading Limited Company. 6727 Szeged, \\ Irinyi J. u. 1. Hungary \\ ${ }^{2}$ Department of Optics and Quantum Electronics, University of Szeged, 6720 Szeged Dóm tér \\ 9. Hungary \\ ${ }^{3}$ MTA-SZTE Research Group on Photoacoustic Spectroscopy, 6720 Szeged Dóm tér 9. \\ Hungary \\ * Corresponding author: tnikolett@titan.physx.u-szeged.hu , Tel: +36-62-544518
}

\begin{abstract}
This paper describes a membrane permeability measuring set-up based on photoacoustic spectroscopy using continuous carrier gas flow to transport the permeated analyte molecules into a photoacoustic detection cell. The permeability parameters of the sample were determined from the measured permeation curves by using a numerical curve fitting algorithm. The method was applied to different membrane samples for determining methane and carbon-dioxide permeability at various carrier gas flow rates (CFRs). For each sample, a characteristic threshold flow rate (TFR) value can be identified below which a strong dependency of the determined permeation parameters on the CFR was found. For those cases when the CFR value cannot be set to be sufficiently high (i.e. above the TFR value), an extrapolation method was presented giving an accurate estimation of the permeation parameters.
\end{abstract}

Keyword: photoacoustics, membrane, permeability, polyethylene, rubber 


\section{Introduction}

Membranes and foils are increasingly used in several areas of science and technology as well as in everyday life. Examples include packaging materials for food and the pharmaceutical industry, where both gas barrier and breathing foils have widespread applications, and the membrane based gas separation method where membranes with gas specific permeability parameters are used [1-4]. Accordingly, permeation measurement methods are increasingly challenged by the wide variety of membranes being measured, as the permeation process is completed on a time scale from seconds to months. Consequently, the detection unit of a general purpose permeation measuring instrument is required to be capable of measuring the permeated molecules over a wide concentration range, with short response time and outstanding long-term stability. Furthermore, in the case of measuring the permeation of a gas mixture, the detection method also has to be selective.

A photoacoustic detection unit can meet all these requirements $[5,6]$. The volume of a photoacoustic detection cell can be as small as a few $\mathrm{cm}^{3}$; thus has a response time in the range of a few seconds [7]. Since the photoacoustic method has a concentration measurement range of 5 to 6 orders of magnitude, it is able to follow the entire permeation independent of the time scale of the process. It has to be emphasized that the photoacoustic method is based on laser spectroscopy; therefore it is also applicable in selective permeation measurements[8]. In a previous paper, the authors introduced a closed loop carrier gas circulation based photoacoustic detection system for measuring barrier membranes with low permeability [9]. In the work reported here, an alternative measurement set-up is presented which, to the best of the author's knowledge, is the first time where the photoacoustic detection unit is operated in a carrier gas flow based arrangement. It is motivated by our previous studies where it was proved that photoacoustics is a viable alternative of direct optical absorption based concentration measuring methods [10-11].

In a carrier gas flow based permeation measuring system, the membrane is placed in a diffusion cell such that it divides the cell into two hermetically separated parts, into a source and a receiving chamber [12-13]. At the start of the measurement, the source chamber is flushed with the feed gas containing the measured analyte in high (typically 100\%) concentration, while the receiving chamber is continuously purged with the carrier gas that originally does not contain the permeating component. Molecules diffusing through the membrane gradually increase the concentration of the permeating component in the receiving chamber, which is measured by a detection unit where the permeated molecules are transferred by the carrier gas. 
For many types of membranes, the permeation process can be accurately described by a simple one dimensional partial differential equation based on Henry's and Fick's laws, while the gas permeability of the studied membrane can be fully characterised by concentration independent diffusivity $(D)$ and solubility $(S)$ constants [14]. However, this simple description is valid only if all of the following experimental conditions are met:

1. At the initiation of the permeation process the time needed for the build-up of the high concentration of the analyte in the source chamber is negligible compared to the characteristic time scale of the permeation process.

2. The diffusion takes place only through the thickness of the membrane and, consequently, the permeation process can be described mathematically as being onedimensional.

3. The time needed to transfer the analyte molecules from the receiving chamber into the detection unit is negligible compared to the characteristic time scale of the permeation process.

In most cases, the flow rate of the feed gas can be increased up to a sufficiently high value, resulting in a quasi-instantaneous build-up of the high analyte concentration in the source chamber. As far as the second requirement is concerned, the permeation process can be restricted to be quasi one-dimensional by performing permeation measurement on a membrane having lateral dimensions at least an order of magnitude larger than its thickness. However, meeting the third assumption can often be difficult.

In the work presented here, a carrier gas based permeation measuring instrument containing a photoacoustic detection unit is introduced, the effect of the limits on the applicable carrier gas flow rate on the deduced permeation parameters is evaluated and a method is suggested for extending the range of measurable permeation parameters. 


\section{Experimental}

\subsection{Experimental setup}

The developed permeation measuring system shown schematically in Figure 1 consists of the diffusion cell (DC), the photoacoustic detection cell (PAC) and the gas handling system. The membrane to be measured is placed in the cylindrical shaped diffusion cell with internal height of $2.5 \mathrm{~cm}$ and diameter of $7 \mathrm{~cm}$. The membrane divides the cell into two hermetically separated parts: receiving chamber with $0.5 \mathrm{~cm}$ internal height (having a volume of $19.2 \mathrm{~cm}^{3}$ ) and source chamber with $2.0 \mathrm{~cm}$ internal height (volume of $77 \mathrm{~cm}^{3}$ ) [12-14]. Each chamber has inlet and outlet gas ports. The source chamber is purged either with the carrier gas (for cleaning it from the previous measurement) or with the feed gas. Switching between these gases is made by a three port switching solenoid valve (MV) (VDW250, SMC Pneumatics, Inc.) controlled by the electronics. The receiving chamber is purged with the carrier gas, which is subsequently fed into the photoacoustic detection cell. The flow rate can be set between 20-1000 standard cubic centimetres per minute ( $\mathrm{sccm})$ in the source chamber and 10$500 \mathrm{sccm}$ in the receiving chamber, using two thermal mass flow controllers (MFC) (SLA5850S, Brooks Instrument, LLC).

The photoacoustic detection cell is a longitudinal differential cell with an inner gas volume of approximately $20 \mathrm{~cm}^{3}$ which operates with continuous gas flow at a maximum flow rate of $500 \mathrm{sccm}$. Above this limit, the minimum detectable concentration increases sharply due to the noise of the measurement caused by the acoustic noise of the flow generated in the photoacoustic cell. The special differential construction of the cell resulted in this maximum allowable flow rate being actually among the highest reported in the literature for various types of photoacoustic detection cells [15]. For methane and for carbon-dioxide detection, two telecommunication type fibre-coupled single emission mode diode lasers are used with wavelength of $1652 \mathrm{~nm}$ (DL) (NLK1U5FAAA, NEL Electronics Corp.) and 1574 nm (FOL15DCWD, Furukawa Electric Co., LTD.), respectively. The signal from the microphones (Knowles EK-3029) is amplified and processed by the electronics (developed by the Development Institute of Videoton Holding Plc.) using a digital lock-in technique. Additionally, the electronics generates driving current for the lasers, stabilizes the temperature of the lasers and the detection cell, and drives the mass flow controllers and the magnetic valves [16]. The methane and carbon-dioxide concentrations are also calculated by the electronics based on preparatory calibrations. As is indicated in Figure 1, one unique advantage of the photoacoustic method is that up to three detection cells can be placed one 
after the other, in a way that the same laser beam propagates through them making it possible to perform permeation measurements on up to three samples simultaneously.

The minimum detectable methane and carbon-dioxide concentration in the presented setup was about 0.5 and $50 \mathrm{ppm}$, respectively. The maximum measurable concentration was practically $100 \%$ for both analytes.

\subsection{Materials}

The carrier gas was nitrogen (99.9995\% purity), the feeding gas was either $\mathrm{CH}_{4}(99.995 \%$ purity) or $\mathrm{CO}_{2}$ (99.9995\% purity). The following membranes were measured:

- Polyethylene (PE) (Tradeplast Ltd.). Type 1: $0.14 \mathrm{~mm}$ thick, permeating analytes were $\mathrm{CH}_{4}$ and $\mathrm{CO}_{2}$; type 2: $0.06 \mathrm{~mm}$ thick, permeating analyte was $\mathrm{CH}_{4}$.

- Silicone rubber (Trelleborg, $\mathrm{AB}$ ): $2.5 \mathrm{~mm}$ thick, permeating analytes were $\mathrm{CH}_{4}$ and $\mathrm{CO}_{2}$.

- Natural rubber sheets (Contitech Rubber Industrial Ltd.): $0.7 \mathrm{~mm}$ thick, permeating analyte was $\mathrm{CH}_{4}$.

\subsection{The measurement procedure}

Measurement started with purging both parts of the permeation cell and the detection unit with the carrier gas. After that, the magnetic valve was switched and the source chamber was purged with the feed gas while the receiving chamber was still fed with the carrier gas. The concentration of the permeated analyte in the photoacoustic cell was recorded. The measurement procedure was repeated for different samples and feed gases at various CFRs.

\subsection{Data evaluation}

The permeation process can be described by the permeation curve which is a graph showing $Q(t)$ as a function of time. $Q(t)$ represents the amount of analyte permeated through the unit surface area of the membrane since the start of the permeation process. $Q(t)$ can be calculated from the measured analyte concentration $(C(t))$ with the following equation:

$$
Q(t)=\int_{0}^{t} \frac{C(\tau) \cdot v}{A} d \tau
$$

where $v$ is the CFR value and $A$ is the surface area of the membrane. The lower limit of this integration is the time when the source chamber is purged by the feed gas, while the upper limit is the actual time. 
Under ideal circumstances, the permeation curve obeys the following formula:

$$
Q(t)=l \Delta p S\left[\frac{D t}{l^{2}}-\frac{1}{6}-\frac{2}{\pi^{2}} \sum_{n=1}^{\infty} \frac{(-1)^{n}}{n^{2}} \exp \left(-\frac{D n^{2} \pi^{2} t}{l^{2}}\right)\right\rfloor
$$

where $l$ is the thickness of the membrane, $\Delta p$ is the initial partial pressure difference of the permeating analyte between the source and the receiving chamber, $S$ is the solubility and $D$ is the diffusivity of the membrane $[14,17]$.

There are two approaches typically applied for the determination of the permeation parameters from the permeation curve. The first is the so called time-lag analysis method. This is based on the fact that, after an early transient part, the permeation curve has a steady state region during which the total amount of analyte permeated through the sample increases linearly with time. By fitting a line to this steady state part, $D$ and $S$ constants of the sample were determined as:

$$
\begin{gathered}
D=\frac{l^{2}}{6 \theta} \\
S=\frac{N l}{\Delta p D}
\end{gathered}
$$

where $N$ is the slope and $\theta$ is the x-axis intersection of the fitted line. Alternatively, whenever the signal-to-noise ratio of the measurement was high enough, even during the early part of the permeation process when the analyte concentration in the receiving chamber was low, Equation 1 was fitted numerically to the entire permeation curve [9]. The outputs of this numerical fitting algorithm are the $D$ and $S$ constants.

\section{Results}

Methane permeation curves for the PE type-1 sample measured with two different CFRs together with the lines fitted to their steady state parts can be seen in Figure 2. The measured permeation curves and the fitted lines were different, therefore the deduced permeation parameters (marked with the index "app" emphasising the fact that these are apparent, i.e. CFR dependent parameters) differed also. Using either the least square fitting algorithm or the time-lag analysis method, these apparent permeation parameters were $D_{a p p}=1.45 \cdot 10^{-11} \mathrm{~m}^{2} / \mathrm{s}, S_{a p p}=1.32 \cdot 10^{-6} 1 / \mathrm{Pa}$ and $D_{a p p}=1.87 \cdot 10^{-11} \mathrm{~m}^{2} / \mathrm{s}, S_{a p p}=1.03 \cdot 10^{-6} 1 / \mathrm{Pa}$ for CFR values of $50 \mathrm{sccm}$ and $400 \mathrm{sccm}$, respectively.

In Figure 3a-c, apparent permeation parameters calculated by the least square fitting algorithm on measurements performed with various CFR values can be seen. While the 
apparent permeation parameters have strong dependency on the CFR values at low flow rates, a TFR value can be clearly identified above which the permeation parameters become virtually independent of the CFR. For measurements shown in Figure $3 a$ and $3 b$, the TFR value can be estimated to be $200 \mathrm{sccm}$ and $80 \mathrm{sccm}$, respectively. As far as the measurements shown in Figure 3c are concerned, no TFR value can be identified from the figure, probably because it was higher than the maximum allowable flow rate through the photoacoustic cell (i.e. $500 \mathrm{sccm}$ ). Error bars indicate standard deviation of 5 independent measurements.

\section{Discussion}

As it was emphasized, permeation measurement yields reliable permeation parameters only if the analyte molecules from the receiving chamber are transferred into the detection unit quasi-instantaneously. Accordingly, carrier gas flow rate independent permeation constants $(D$ and $S$ ) can be determined only if the permeation measurements are performed with CFR value above a certain limit (i.e. the TFR value) specific to the measured sample and the measurement conditions (Figure 3). This raises further challenges; since the permeation measurement has to be performed at a sufficiently high CFR value rather than repeating the measurements at various flow rates to verify that the determined permeation parameters are flow rate independent. In addition, the permeation parameters have to be determined with sufficient accuracy, even if the measurement cannot be performed at the required CFR value as it is actually higher than the maximum flow rate allowed by the measurement set-up. Both of these problems can be solved by using a new, sample and measurement condition specific parameter, termed Carrying Efficiency $(C E)$, defined as:

$$
C E=\frac{l^{2} v}{6 D_{a p p} V_{t o t}}
$$

where $v$ is the actual value of the CFR and $V_{\text {tot }}$ is the total volume of the receiving chamber, the photoacoustic cell and the tubing in-between them. $C E$ can be interpreted as the total volume of the carrier gas which flows through the detection cell during the time interval $\theta$ (see Equation 2) divided by the total volume of the receiving side of the set-up. In Figure $4 \mathrm{a}$ and $\mathrm{b}$, calculated apparent permeation parameters for different samples and permeating analytes, at various CFR values are plotted against corresponding $C E$ values in a way that the permeation parameters are normalised with their CFR independent value measured at sufficiently high flow rate. Figure 4 suggests the existence of general trend curves which were determined by curve fitting as: 


$$
\begin{gathered}
D_{a p p}=D\left(1-0.92 \cdot e^{-\frac{C E}{2.85}}\right) \\
S_{a p p}=S\left(1+1.9 \cdot e^{-\frac{C E}{2.57}}\right)
\end{gathered}
$$

Based on Equation 5 and 6, the apparent permeation parameters differ less than 5\% from their true values whenever $C E \geq 10$. The flow rate corresponding to $C E=10$ can be considered to be the TFR value, given as:

$$
T F R=\frac{60 D_{a p p} V_{t o t}}{l^{2}}
$$

This equation can be used in data evaluation as follows. After completing a permeation measurement, $D_{a p p}$ and the corresponding TFR value can be calculated. If the CFR value used in the measurement is actually higher than this TFR value, the apparent permeation parameters can be expected to differ from their true values by no more than $5 \%$, which is generally considered to be good accuracy for permeation measurements. Otherwise, from the measured apparent permeation parameters, $D$ and $S$ can be extrapolated using Equations 4-6. Obviously, there is also the possibility of validating the extrapolated values by repeating the measurement with a sufficiently high CFR value, unless the measurement conditions prohibit this verification (i.e. CFR cannot be set to be higher than the TFR value, see below).

Results of the performed permeation measurements are summarized in Table 1. These are calculated from the results of measurements where the $C E$ value was higher than 10 , with the exception of the two measurements marked with an asterisk. In the case of $\mathrm{CH}_{4}$ permeation measurement on PE type-2 sample, the measurements had to be performed at CFR values lower than the TFR, because the TFR value was found to be higher than the maximum allowed flow rate through the photoacoustic detection cell. In the case of $\mathrm{CO}_{2}$ permeation measurement on the PE type-1 sample, the concentration measurements with CFR values higher than the TFR value were found to have prohibitively low signal to noise ratio and correspondingly high uncertainty. This is the consequence of the dilution effect of the carrier flow, i.e. that the analyte concentration in the detection cell is inversely proportional to the CFR, therefore increasing the flow rate decreases the signal to noise ratio of the concentration measurement. This effect generally occurs in a carrier gas flow arrangement regardless of the detection method. For these two cases, the determination of the permeation parameters was based on the suggested extrapolation method. There is a good agreement between the $D$ and $S$ 
constants listed in Table 1 and data from the literature [18], although a rigorous comparison is not really possible due to potential differences in the measured samples.

\section{Conclusions}

The aim of the presented work was to introduce photoacoustics as a detection method in carried gas flow based permeation measurements as an alternative of the commonly used infrared absorption sensors. There are multiple advantages of using the photoacoustic detection method for permeability measurements which includes the wide range of measurable concentrations, short response time and excellent selectivity of the measurement, as well as the possibility of measuring multiple samples simultaneously. Based on the photoacoustic detection method, permeation parameters of various samples were determined and they were in good agreement with the literature values.

Furthermore, a simple method is also suggested to extrapolate permeation parameters from measurements performed with insufficiently high CFRs restricted either by the flow noise sensitivity of the photoacoustic detection cell or by the dilution effect of the carrier gas on the permeated molecules. The extrapolation method proved to have a good accuracy.

It should be noted that, while the diffusivity and solubility constants show strong dependence on the CFR at low flow rates, permeability is far less dependent on the flow rate as $t$ could be expected from the converse shape of the trend curves in Figure $4 \mathrm{a}$ and $\mathrm{b}$.

Finally, the introduced extrapolation method based on the carrier efficiency parameter is expected to be applicable for carrier gas flow based permeation measurements using not only photoacoustics but also other detection methods.

\section{Acknowledgement}

The financial support of the TAMOP-4.2.2.A-11/1/KONV-2012-0047 project is acknowledged.

\section{References}

1. R.E. Kesting. Synthetic polymeric membranes. 2nd ed. John Wiley and Sons, New York. 1985

2. C.A. Scholes, G.W. Stevens, S.E. Kentish. Membrane gas separation applications in natural gas processing. Fuel. 2012; 96:15-28.

3. Baker R.W. Membrane technology and applications. 2nd ed. John Wiley and Sons, Chichester. 2004 
4. J. Comyn. Polymer Permeability. 1st ed. Chapman and Hall, London. 1985

5. A. Boschetti, D. Bassi, E. Iacob, S. Iannotta, L. Ricci, M. Scotoni. Resonant photoacoustic simultaneous detection of methane and ethylene by means of a $1.63-\mu \mathrm{m}$ diode laser. Appl. Phys. B 2002; 74(3):273-278.

6. A. Elia, P.M. Lugarà, C. Di Franco, V. Spagnolo. Photoacoustic Techniques for Trace Gas Sensing Based on Semiconductor Laser Sources. Sensors 9. 2009; 12:9616-9628.

7. Z. Bozóki, A. Szabó, Á. Mohácsi, G. Szabó. A fully opened photoacoustic resonator based system for fast response gas concentration measurements. Sensor. Actuator. B Chem., 2010; 147(1): 206-212.

8. M. Al-Ismaily, J.G. Wijmans, B. Kruczek. A shortcut method for faster determination of permeability coefficient from time lag experiments. J. Membr. Sci.. 2012; 165-174.

9. Z. Filus, T. Ajtai, Z.L. Horváth, Z. Bozóki, G. Pap, T. Nagy, T. Katona, G. Szabó. A novel apparatus based on a photoacoustic gas detection system for measuring permeation parameters of polymer samples. Polym. Test. 2007; 26(5):606-613.

10. Z. Bozóki, Á. Mohácsi, G. Szabó, Z. Bór, M. Erdélyi, W. Chen, F. K. Tittel. Near infrared diode laser based spectroscopic detection of ammonia: a comparative study of photoacoustic and direct optical absorption methods. Appl. Spectrosc. 2002; 56:715-719.

11. T. Ajtai, Á. Filep, A. Varga, G. Motika, Z. Bozóki, G. Szabó. Ozone concentration monitoring photoacoustic system based on a frequency quadrupled Nd:YAG laser. Appl. Phys. B. 2010; 101:403-409.

12. ISO 15105-2, Plastics - Film and sheeting - Determination of gas-transmission rate Part 2: Equal-pressure method

13. ISO 15106-2, Plastics - Film and sheeting - Determination of water vapour transmission rate - Part 2: Infrared detection sensor method

14. J. Crank. The Mathematics of Diffusion. 2nd ed. Clarendon Press, Oxford. 1975

15. A. Miklós, P. Hess, Z. Bozóki. Application of acoustic resonators in photoacoustic trace gas analysis and metrology. Rev. Sci. Instrum. 2001; 72(4):1937-1955.

16. A. Varga, Z. Bozóki, M. Szakáll, G. Szabó. Photoacoustic system for on-line process monitoring of hydrogen sulfide $\left(\mathrm{H}_{2} \mathrm{~S}\right)$ concentration in natural gas streams. Appl. Phys. B. 2006; 85:315-321.

17. B. Flaconneche, J. Martin, M.H. Klopffer. Transport properties of gases in polymers: Experimental methods. Oil Gas Sci. Technol. - Rev. IFP. 2001; 56(3):245-259.

18. J. Brandrup, E.H. Immergut, E.A. Grukle, D. Bloch (Eds.). Polymer Handbook. 4th ed. John Wiley \& Sons, New York. 1999 


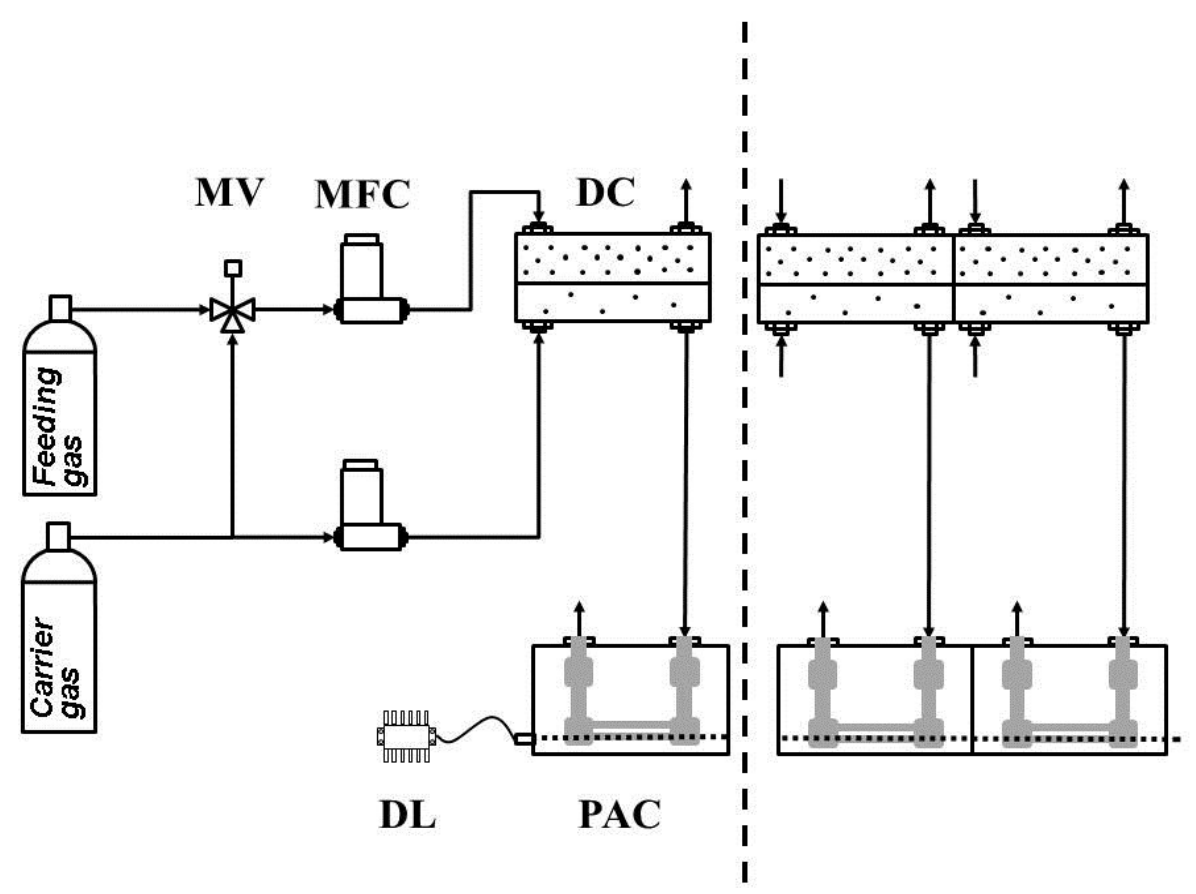

Figure 1. Schematics of the permeation measuring set-up based on carrier gas flow and photoacoustic detection. DC: diffusion cells, MFC: mass flow controllers, PAC:

photoacoustic detection cells, DL: diode-laser, MV: three way magnetic valve. The possibility of upgrading the set-up to measure up to three membrane samples simultaneously by using the same light source is also indicated.

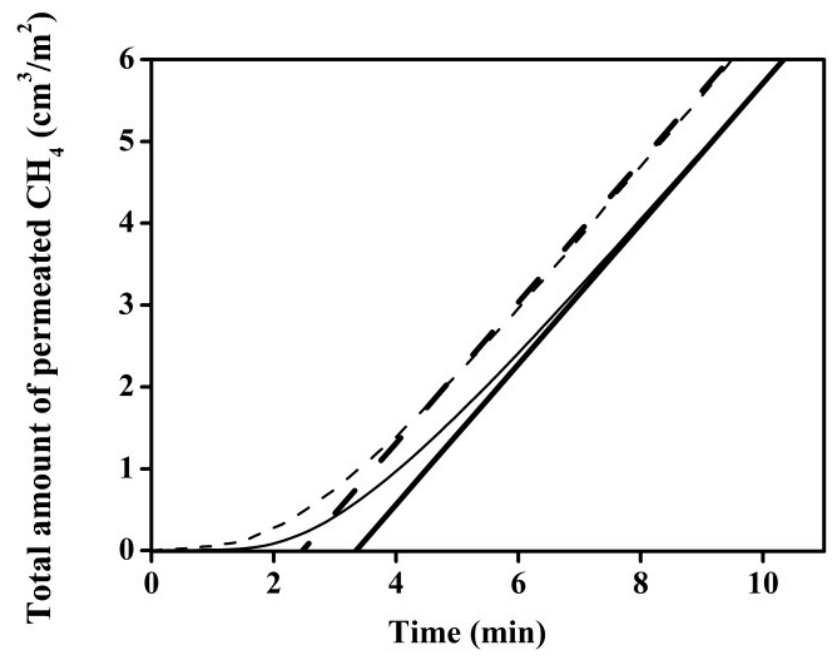

Figure 2. Methane permeation curves of PE type-1 sample measured at carrier gas flow rate of $50 \mathrm{sccm}$ (thin solid line) and $400 \mathrm{sccm}$ (thin dash line). Lines fitted to the steady-state parts of the curves are shown as well (thick lines). 


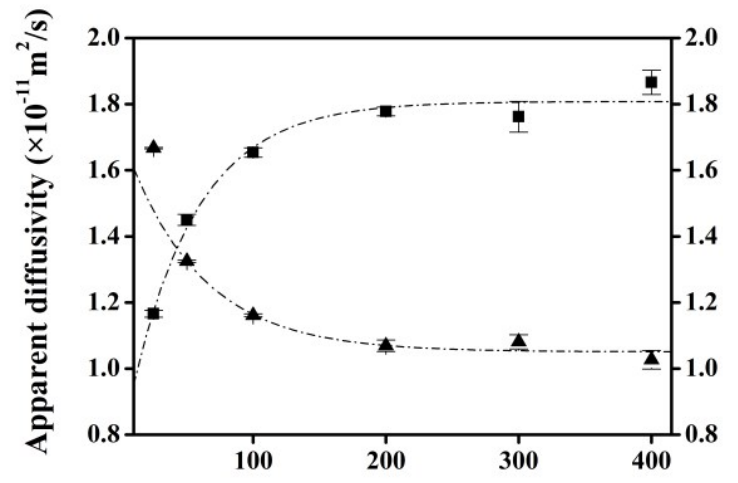

$\mathbf{a}$

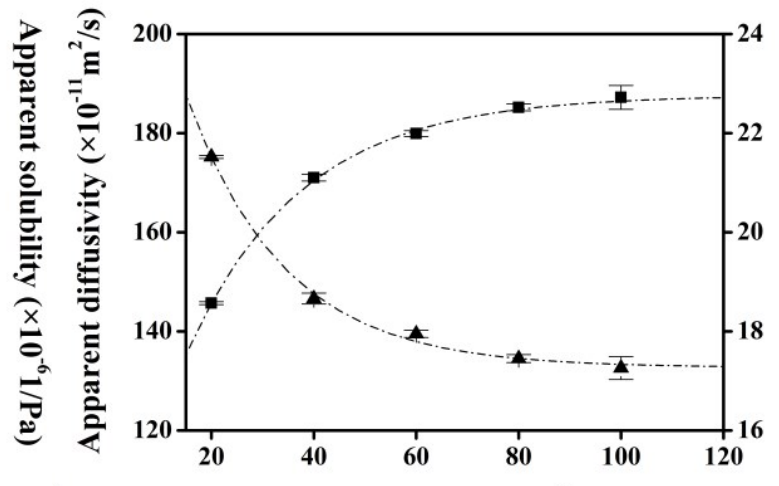

b

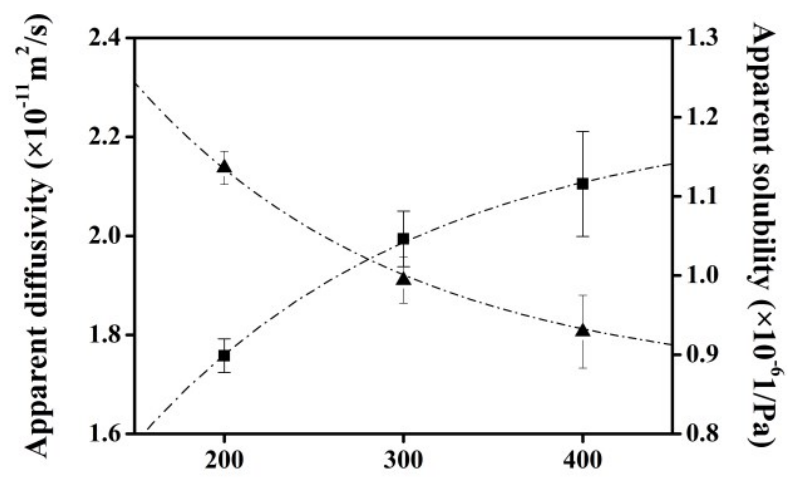

Carrier flow rate $\left(\mathrm{cm}^{3} / \mathrm{min}\right)$

Figure 3. Carrier gas flow rate dependence of the apparent permeation parameters a: PE type-1 and methane, b: silicone rubber and carbon-dioxide c: PE type-2 and methane. Dapp (squares) and Sapp (triangles) for different sample-gas combinations Trend lines are drawn to guide the eye.
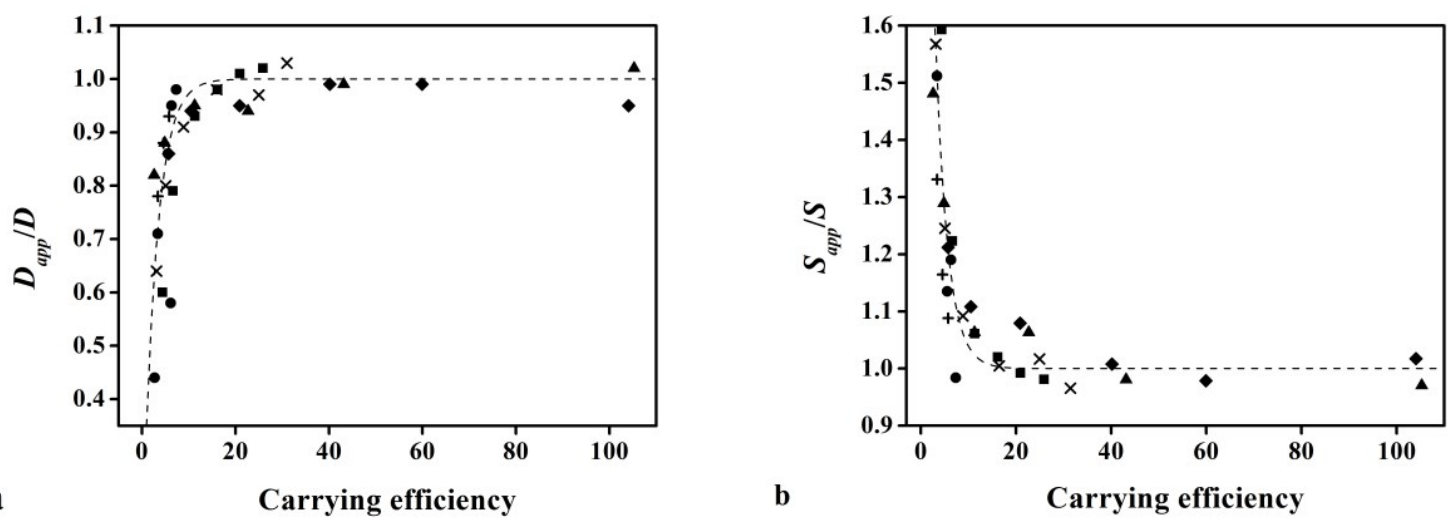

Figure 4. Carrying efficiency dependence of the apparent permeation parameters normalised with their optimum values for the following samples and permeating analytes; PE type-1 and $\mathrm{CH}_{4}(\times)$, PE type-2 and $\mathrm{CH}_{4}(+)$, silicone rubber and $\mathrm{CH}_{4}(\diamond)$, natural rubber and $\mathrm{CH}_{4}(\boldsymbol{\Delta})$, PE type-1 and $\mathrm{CO}_{2}(\bullet)$ and silicone rubber and $\mathrm{CO}_{2}(\boldsymbol{\bullet})$. Numerically fitted trend curves are shown as dashed lines. 
Table 1

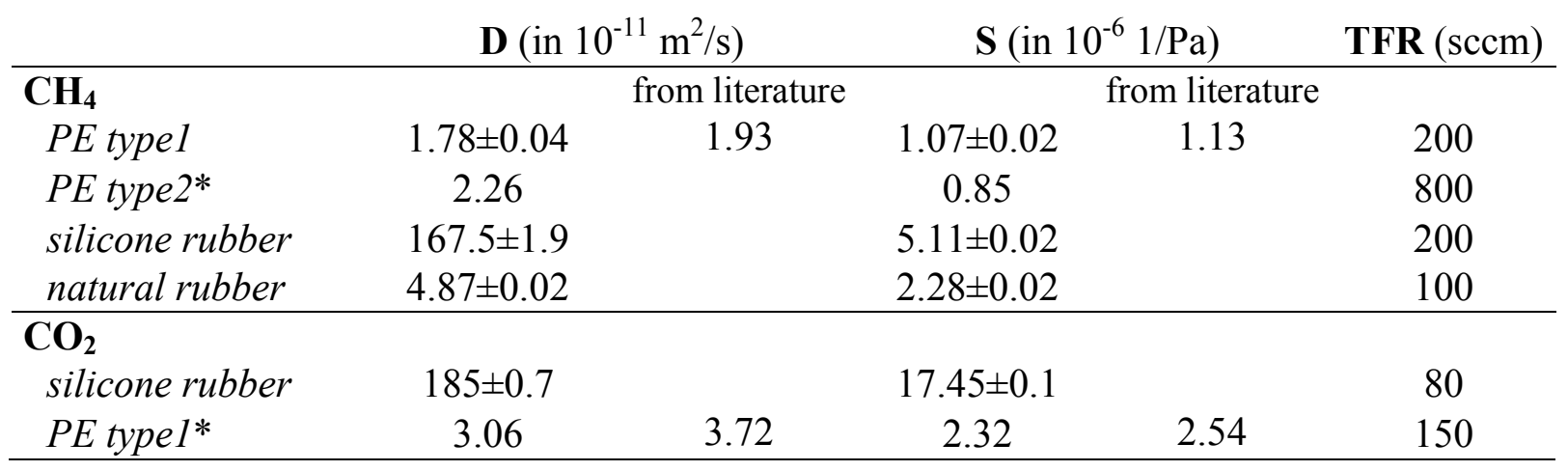

Table 1. List of D, S and TFR constants determined for different samples and permeating analytes. Those measurements where these parameters were determined via extrapolation are marked with asterisks. 